\title{
Fiber-homotopy self-equivalences and a classification of fibrations in rational homotopy
}

\author{
Toshihiro Yamaguchi $^{1}$ - Shoji Yokura ${ }^{2}$
}

Received: 6 April 2015 / Accepted: 28 September 2015 / Published online: 17 October 2016

(C) Tbilisi Centre for Mathematical Sciences 2016

\begin{abstract}
For a fibration $\xi: X \stackrel{i}{\rightarrow} E \stackrel{p}{\rightarrow} B$ we consider the image of the rationalized homotopy group homomorphism $\pi_{*}(\operatorname{res} \xi)_{\mathbb{Q}}: \pi_{*}\left(\operatorname{aut}_{1} \mathrm{p}\right)_{\mathbb{Q}} \rightarrow \pi_{*}\left(\text { aut }_{1} \mathrm{X}\right)_{\mathbb{Q}}$ obtained from the fibre-restricting map res $\xi:$ aut $_{1} \mathrm{p} \rightarrow$ aut $_{1} X$. Then we consider a finite type classification of fibrations $\xi$ with fibre $X$ and base $B$. In particular, we measure the size of it by a rational homotopical invariant " $\operatorname{depth}_{B} \mathrm{X}$ " when $X$ are certain homogeneous spaces and $B$ are spheres.
\end{abstract}

Keywords Fibre-homotopy self-equivalences · Rational homotopy · Sullivan minimal model $\cdot$ Pure space - Derivation · Totally non-cohomologus to zero (tncz) · Halperin conjecture $\cdot$ Depth

\section{Introduction}

In this paper, a fibration $\xi: X \stackrel{i}{\rightarrow} E \stackrel{p}{\rightarrow} B$ is a Hurewicz fibration of simply connected spaces. Gottlieb posed the following problem in [12, §5]: Which homotopy equivalences of $X$ into itself can be extended to fibre homotopy equivalences of $E$ into itself?.

Dedicated to Ronnie Brown for his eightieth birthday

Communicated by Tim Porter and George Janelidze.

Toshihiro Yamaguchi

tyamag@kochi-u.ac.jp

Shoji Yokura

yokura@sci.kagoshima-u.ac.jp

1 Faculty of Education, Kochi University, 2-5-1, Kochi 780-8520, Japan

2 Department of Mathematics and Computer Science, Graduate school of Science and Engineering, Kagoshima University, 1-21-35, Korimoto, Kagoshima 890-0065, Japan 
Let aut $X$ be the space of unpointed homotopy self-equivalences of $X$ and let aut $p$ be the space of unpointed fibre-homotopy self-equivalences of $p$, i.e. the subspace of aut $E$ consisting of $g: E \rightarrow E$ satisfying $p \circ g=p$. Then the above Gottlieb's problem is nothing but the problem of determining the image of the "fiber-restricting" map

$$
\operatorname{res} \xi: \text { aut } p \rightarrow \text { aut } X
$$

which is defined by restricting the fiberwise map $f: E \rightarrow E$ to the fiber $X$; $\left.f\right|_{X}: X \rightarrow X$. This problem is replaced as follows: Which homotopy classes of homotopy equivalences of $X$ into itself can be extended to the homotopy class of fibre homotopy equivalences of $E$ into itself? In other words, it is the problem of determining the image of the $\pi_{0}$-version of the map above:

$$
\pi_{0}(\operatorname{res} \xi): \pi_{0}(\text { aut } p) \rightarrow \pi_{0}(\text { aut } X) \text {. }
$$

Here we note that in our previous work [17], using this map $\pi_{0}(\operatorname{res} \xi): \pi_{0}($ aut $p) \rightarrow$ $\pi_{0}($ aut $X)$ for the base space $B=K(\mathbb{Z}, 2)$, we consider a certain poset structure on $X$.

It is quite natural to think of a more general $\pi_{n}$-version of the homomorpism above, i.e. for $n>0$

$$
\pi_{n}(\operatorname{res} \xi): \pi_{n}\left(\operatorname{aut}_{1} p\right) \rightarrow \pi_{n}\left(\operatorname{aut}_{1} X\right)
$$

where aut $_{1} p$ is the path component of aut $p$ containing $i d_{E}: E \stackrel{=}{\rightarrow} E$ and similarly for aut $_{1} X$.

In this paper, first, we discuss the surjectivity of the following rationalized homomorphisms of the total homotopy groups:

$$
\pi_{*}(\operatorname{res} \xi)_{\mathbb{Q}}: \pi_{*}\left(\operatorname{aut}_{1} p\right)_{\mathbb{Q}} \rightarrow \pi_{*}\left(\operatorname{aut}_{1} X\right)_{\mathbb{Q}}
$$

Here $\pi_{*}\left(\operatorname{aut}_{1} p\right)_{\mathbb{Q}}=\bigoplus_{n>0} \pi_{n}\left(\operatorname{aut}_{1} p\right) \otimes \mathbb{Q}$ and $\pi_{*}\left(\operatorname{aut}_{1} X\right)_{\mathbb{Q}}=\bigoplus_{n>0} \pi_{n}\left(\operatorname{aut}_{1} X\right) \otimes \mathbb{Q}$. (We denote $(-) \otimes \mathbb{Q}$ by $(-) \mathbb{Q}$ using the suffix). This (homotopical) surjectivity problem is motivated by the following definition introduced by H. Samelson in early 1940's (cf. [23]). For a fibration $X \stackrel{i}{\rightarrow} E \stackrel{p}{\rightarrow} B$ the fiber $X$ is totally non-cohomologous to zero in $E$ with respect to $G$, where $G$ is a field or the integers $\mathbb{Z}$, if the induced homomorphism $i^{*}: H^{*}(E, G) \rightarrow H^{*}(X, G)$ is surjective. This (cohomological) surjectivity problem has been studied by many people (e.g. $[3,4,18,20,28]$ around 1950 's and recently $[2,21]$, etc.). If $G$ is the rationals $\mathbb{Q}$, we just simply say totally non-cohomologous to zero ( $a b b r$, tncz). This is equivalent to the Leray-Serre spectral sequence of the fibration degenerating on the $E_{2}$-term, and also it is well-known that $H^{*}(X, \mathbb{Q})$ has no negative degree derivations if and only if $X$ is tncz for any fibration over a simply connected base space (equivalently, it holds over an odd-sphere [21]). It is a conjecture due to Halperin ([9]) that if $X$ is an $F_{0}$-space, i.e. it has positive Euler characteristic and $H^{*}(X, \mathbb{Q})$ and $\pi_{*}(X)_{\mathbb{Q}}$ are both finite dimensional (if we require only the second condition, then $X$ is called elliptic), then $X$ is tncz for any fibration. 
Due to Meier [22], it is equivalent to $H^{*}\left(B\right.$ aut $\left._{1} X ; \mathbb{Q}\right)$ being a $\mathbb{Q}$-polynomial algebra for the Dold-Lashof classifying space $B$ aut $_{1} X$ [6]. In 1987, Shiga and Tezuka [30] showed that the Halperin conjecture is true when $X$ is a homogeneous space $G / H$ of $\operatorname{rank} G=\operatorname{rank} H$. In $\S 3$, we consider a correspondence between this condition and the surjectivity of $\pi_{*}(\operatorname{res} \xi) \mathbb{Q}$, for a more general space called a pure space (see Definition 3.1) defined by Sullivan minimal model [9] (see §2).

Next, we propose a kind of coarse way to classify fibrations using (subgroups of) $\pi_{*}\left(\text { aut }_{1} X\right)_{\mathbb{Q}}$. Any fibration $\xi: X \rightarrow E \rightarrow B$ is a pull-back of the universel fibration with fiber $X: X \rightarrow B$ aut $_{1}^{*} X \rightarrow B$ aut $_{1} X$, where aut* $X$ is the subspace (also subgroup) of aut ${ }_{1} X$, consisting of base point preserving self-equivalence maps. Therefore the standard classification of fibrations with fiber $X$ and base $B$ is given by the homotopy set $\left[B, B\right.$ aut $\left._{1} X\right]$. However it is in general hard to analyze. Refer [7,29] for the rational classifications of fibrations using deformation.

We observe that if a fibration $\xi$ with fiber $X$ and base $B$ is fiber homotopic to the trivial fibration $\mathbb{1}: X \rightarrow B \times X \rightarrow B$, then $\operatorname{Im}\left(\pi_{*}(\operatorname{res} \xi)_{\mathbb{Q}}\right)=\operatorname{Im}\left(\pi_{*}(\operatorname{res} \mathbb{1})_{\mathbb{Q}}\right)=$ $\pi_{*}\left(a u t_{1} X\right) \mathbb{Q}$. Then we make

Definition Two fibrations $\xi_{1}$ and $\xi_{2}$ are equivalent if

$$
\operatorname{Im}\left(\pi_{*}\left(\operatorname{res} \xi_{1}\right)_{\mathbb{Q}}\right)=\operatorname{Im}\left(\pi_{*}\left(\operatorname{res} \xi_{2}\right)_{\mathbb{Q}}\right)
$$

This approach is motivated by the previous work [33] of the first named author. Using the base point $b$ we have the evaluation map $\omega:$ aut $_{1} X \rightarrow X$ defined by $\omega(h):=$ $h(b)$ for $h \in$ aut $_{1} X$, thus the above map $\pi_{*}(\operatorname{res} \xi)_{\mathbb{Q}}: \pi_{*}\left(\operatorname{aut}_{1} p\right)_{\mathbb{Q}} \rightarrow \pi_{*}\left(\operatorname{aut}_{1} X\right)_{\mathbb{Q}}$ can be extended to the following sequence:

$$
\pi_{*}(\omega \circ \operatorname{res} \xi)_{\mathbb{Q}}: \pi_{*}\left(\operatorname{aut}_{1} p\right)_{\mathbb{Q}} \stackrel{\pi_{*}(\operatorname{res} \xi)_{\mathbb{Q}}}{\longrightarrow} \pi_{*}\left(\operatorname{aut}_{1} X\right)_{\mathbb{Q}} \stackrel{\pi_{*}(\omega)_{\mathbb{Q}}}{\longrightarrow} \pi_{*}(X)_{\mathbb{Q}} \cdot
$$

Then we have $\operatorname{Im}\left(\pi_{*}(\omega \circ \operatorname{res} \xi)_{\mathbb{Q}}\right) \subset \operatorname{Im}\left(\pi_{*}(\omega)_{\mathbb{Q}}\right) \subset \pi_{*}(X)_{\mathbb{Q}}$. Here $\operatorname{Im}\left(\pi_{n}(\omega)\right)$ is a well-known group called the $n$-th Gottlieb group of $X$, denoted by $G_{n}(X)$, introduced by Gottlieb $[12,13]$. In this sense, $\operatorname{Im}\left(\pi_{n}(\omega \circ \operatorname{res} \xi)\right)$ is named the $n$-th fiber-restricted Gottlieb group of $X$ with respect to the fibration $\xi$ and denoted by $G_{n}^{\xi}(X)$ in [33]. Two fibrations $\xi_{1}$ and $\xi_{2}$ are said to be $\mathbb{Q}$-Gottlieb-group-equivalent provided that $G_{*}^{\xi_{1}}(X)_{\mathbb{Q}}=G_{*}^{\xi_{2}}(X)_{\mathbb{Q}}$. In [24,33], a certain poset on $X$ is introduced by using the inclusion of the fiber-restricted Gottlieb groups. This $\mathbb{Q}$-Gottlieb-groupequivalence of fibrations is coarser because the Gottlieb group $G_{n}(X)_{\mathbb{Q}}$ is identified as a subgroup of $\pi_{n}\left(\operatorname{aut}_{1} X\right)_{\mathbb{Q}}$. See Example 5.1 that $G_{*}(X)_{\mathbb{Q}}=G_{*}^{\xi}(X)_{\mathbb{Q}}$ but $\pi_{*}\left(\operatorname{aut}_{1} X\right)_{\mathbb{Q}} \neq \operatorname{Im}\left(\pi_{*}(\operatorname{res} \xi) \mathbb{Q}\right)$.

In $\S 4$, we introduce a poset $\mathcal{P}_{B}(X)$ on $X$ using the inclusions of the subgroups $\operatorname{Im}\left(\pi_{*}(\operatorname{res} \xi)_{\mathbb{Q}}\right)$ 's of $\pi_{*}\left(\operatorname{aut}_{1} X\right) \mathbb{Q}_{\mathbb{Q}}$ where the total spaces are finite (see Definition 4.2), and consider $\operatorname{depth}_{B}(X)$, which is the height of the Hasse diagram of $\mathcal{P}_{B}(X)$. Also $\operatorname{depth}(X)$ is defined to be the maximum of $\operatorname{depth}_{B}(X)$ where the base space $B$ runs through all simply connected spaces.

From arguments given in $\S 3$ and $\S 4$ we deduce one of our results:

Theorem 1.1 Let $X$ be a homogeneous space $G / H$ with $\operatorname{rank} \pi_{\text {even }}(X)=2$. Then the followings are equivalent. 
(1) $H^{*}($ Baut $1 X ; \mathbb{Q})$ is a polynomial algebra.

(2) $\pi_{*}(\operatorname{res} \xi)_{\mathbb{Q}}: \pi_{*}\left(\operatorname{aut}_{1} p\right)_{\mathbb{Q}} \rightarrow \pi_{*}\left(\text { aut }_{1} X\right)_{\mathbb{Q}}$ is surjective for any fibration $\xi: E \stackrel{p}{\rightarrow}$ $S^{n}$ with fiber $X$.

(3) $\operatorname{depth}_{S^{n}}(X)=0$ for any $n$.

Here we note that the space $X$ satisfying (1)-(3) of Theorem 1.1 may not be an $F_{0^{-}}$ space (i.e. $\operatorname{rank} G=\operatorname{rank} H$ in this case). See Example 3.5, in which there is a fibration $\xi$ such that $i^{*}: H^{*}(E ; \mathbb{Q}) \rightarrow H^{*}(X ; \mathbb{Q})$ is not surjective (i.e. $X$ is not tncz in $E$ ). Furthermore, in $\S 5$, we give some examples of $\operatorname{depth}(X)>0$. In particular, we see a finite type classification of fibrations in Example 5.5. In $\S 2$ we list some tools in order to obtain such results.

\section{Preliminaries: Sullivan models and derivations}

In this section we recall Sullivan models and related facts for later use.

We use the Sullivan minimal model $M(Y)$ of a simply connected space $Y$ of finite type. It is a free $\mathbb{Q}$-commutative differential graded algebra (abbr. CDGA) $(\Lambda V, d)$ with a $\mathbb{Q}$-graded vector space $V=\bigoplus_{i>1} V^{i}$ where $\operatorname{dim} V^{i}<\infty$ and a decomposable differential, i.e.

$$
d\left(V^{i}\right) \subset\left(\Lambda^{+} V \cdot \Lambda^{+} V\right)^{i+1} \text { and } d \circ d=0 .
$$

Here $\Lambda^{+} V$ is the augmentation ideal of $\Lambda V$. We often denote $(\Lambda V, d)$ simply by $\Lambda V$. The degree of an element $x$ of a graded algebra is denoted by $|x|$. Then we have

$$
x y=(-1)^{|x||y|} y x \text { and } d(x y)=d(x) y+(-1)^{|x|} x d(y) .
$$

We note that $M(Y)$ determines the rational homotopy type $Y_{\mathbb{Q}}$ of $Y$. In particular, there are the following isomorphisms

$$
\operatorname{Hom}\left(V^{i}, \mathbb{Q}\right) \cong \pi_{i}(Y)_{\mathbb{Q}} \text { and } H^{*}(\Lambda V, d) \cong H^{*}(Y ; \mathbb{Q})
$$

See [9] for a general introduction and the standard notations.

Let $A=\left(A^{*}, d_{A}\right)$ be a CDGA with $A^{*}=\oplus_{i \geq 0} A^{i}, A^{0}=\mathbb{Q}, A^{1}=0$ and the augmentation $\epsilon: A \rightarrow \mathbb{Q}$. Define $\operatorname{Der}_{i} A$ to be the vector space of derivations of $A$ decreasing the degree by $i>0$, where

$$
\theta(x y)=\theta(x) y+(-1)^{i|x|} x \theta(y) \text { for } \theta \in \operatorname{Der}_{i} A
$$

Here $\operatorname{Der}_{1} A$ is required to be $\delta$-cycles. We denote $\bigoplus_{i>0} \operatorname{Der}_{i} A$ by $\operatorname{Der} A$. The boundary operator $\delta: \operatorname{Der}_{*} A \rightarrow \operatorname{Der}_{*-1} A$ is defined by

$$
\delta(\sigma)=d_{A} \circ \sigma-(-1)^{|\sigma|} \sigma \circ d_{A} .
$$


$\operatorname{Der} A$ is a differential graded Lie algebra (DGL), where the Lie bracket is given by

$$
[\sigma, \tau]:=\sigma \circ \tau-(-1)^{|\sigma||\tau|} \tau \circ \sigma
$$

It is known $[31, \S 11]$ that

$$
\pi_{*+1}\left(B \operatorname{aut}_{1} Y\right) \otimes \mathbb{Q}=\pi_{*}\left(\operatorname{aut}_{1} Y\right) \otimes \mathbb{Q} \cong H_{*}(\operatorname{Der} M(Y), \delta),
$$

which is the case when the base space $B$ is a point in Theorem 2.4 below. In this paper, following [31, p. 314], the symbol $(v, h) \in \operatorname{Der}_{|v|-|h|}(\Lambda V)$ means the derivation sending an element $v \in V$ to $h \in \Lambda V$ and the other to zero. In particular $(v, 1)=v^{*}$. The differential is given by $\delta(v, h)=d \circ(v, h)-(-1)^{|v|-|h|}(v, h) \circ d$. Recall the notation of Tanré [32, p. 25]: $C^{*}(L, \partial):=\left(\Lambda s^{-1} \sharp L, D=d_{1}+d_{2}\right)$ with

$$
\begin{aligned}
\left\langle d_{1} s^{-1} z ; s x\right\rangle & =-\langle z ; \partial x\rangle \text { and } \\
\left\langle d_{2} s^{-1} z ; s x_{1}, s x_{2}\right\rangle & =(-1)^{\left|x_{1}\right|}\left\langle z ;\left[x_{1}, x_{2}\right]\right\rangle
\end{aligned}
$$

for a differential graded Lie algebra $(L, \partial)$. Here $L$ is a positive graded vector space of finite type and $\sharp L$ means the dual space of $L$ with $|s x|=|x|+1$ and $\left|s^{-1} z\right|=|z|+1$. (The symbols $s$ and $s^{-1}$ mean "shifting degree" respectively for $L$ and $\sharp L$.)

Let $X$ be an elliptic space. Then

Theorem 2.1 ([31, p.314]) The DGA $C^{*}(\operatorname{Der} M(X), \delta)$ is the Sullivan (non-minimal in general) model of $B$ aut $_{1} X$.

This Theorem implies the isomorphism 2.1 above. Therefore we have

Corollary 2.2 $H_{\text {even }}(\operatorname{Der} M(X))=0$ if and only if $H^{*}\left(B\right.$ aut $\left._{1} X ; \mathbb{Q}\right)$ is a polynomial algebra.

Even if $X$ is a Lie group of low rank, $H^{*}\left(B\right.$ aut $\left._{1} X ; \mathbb{Q}\right)$ is not free in general (see Example 2.6 below).

Theorem 2.3 ([8]) For $M(Y)=(\Lambda V, d)$ and the augmentation $\epsilon: M(Y) \rightarrow \mathbb{Q}$,

$$
G_{n}(Y)_{\mathbb{Q}} \cong \operatorname{Im}\left(H_{n}\left(\epsilon_{*}\right): H_{n}(\operatorname{Der} M(Y)) \rightarrow \operatorname{Hom}_{n}(V, \mathbb{Q})\right)
$$

for $n>0$.

Félix, Lupton and Smith showed

Theorem 2.4 ([10, Theorem 1.1]) For a fibration $p: E \rightarrow B$ with $E$ and B simply connected and E finite,

$$
\pi_{*}\left(\operatorname{aut}_{1} p\right)_{\mathbb{Q}} \cong H_{*}\left(\operatorname{Der}_{\Lambda V}(\Lambda V \otimes \Lambda W), \delta\right),
$$

where $\left(\Lambda V, d_{B}\right) \rightarrow(\Lambda V \otimes \Lambda W, D)$ is the Koszul-Sullivan model (abbr. KS-model) of $p$ and $\left(\operatorname{Der}_{\Lambda V}(\Lambda V \otimes \Lambda W), \delta\right)$ is the chain complex of derivations vanishing on $\Lambda V$. 
Then we get

Proposition 2.5 ([33]) For a fibration $X \rightarrow E \stackrel{p}{\rightarrow} B$, the map $\pi_{n}(\operatorname{res} \xi)_{\mathbb{Q}}$ : $\pi_{n}\left(\operatorname{aut}_{1}(p)\right)_{\mathbb{Q}} \rightarrow \pi_{n}\left(\operatorname{aut}_{1} X\right)_{\mathbb{Q}}$ is given by the restriction of derivations

$$
R_{n}: H_{n}\left(\operatorname{Der}_{\Lambda V}(\Lambda V \otimes \Lambda W), \delta\right) \rightarrow H_{n}(\operatorname{Der}(\Lambda W))
$$

Example 2.6 When $X$ is the special unitary group $S U(3)\left(\simeq \mathbb{Q} S^{3} \times S^{5}\right)$ and $B=S^{3}$, there is a non-trivial fibration $X \rightarrow E \rightarrow B$ with $D w_{2}=v w_{1}$ and $D w_{1}=0$ for $H^{*}(X ; \mathbb{Q})=\Lambda\left(w_{1}, w_{2}\right)$ with $\left|w_{1}\right|=3$ and $\left|w_{2}\right|=5$ and $H^{*}(B ; \mathbb{Q})=\Lambda v$ with $|v|=3$. Then $\operatorname{Im} R_{*}=\operatorname{Im} R_{2} \oplus \operatorname{Im} R_{5}=\mathbb{Q}\left\{\left(w_{2}, w_{1}\right),\left(w_{2}, 1\right)\right\}$. Notice that the element $\left(w_{1}, 1\right)$ is not a $\delta$-cycle in $\operatorname{Der}_{\Lambda V}(\Lambda V \otimes \Lambda W)$ since $\delta\left(w_{1}, 1\right)=-\left(w_{2}, v\right)$. Thus $R_{*}=\oplus R_{n}$ is not surjective since $\left(w_{1}, 1\right)$ is not in $\operatorname{Im} R_{3}$. From Theorem 2.1 the cohomology of $B$ aut $_{1} X$ is infinitely generated as

$$
H^{*}\left(B \text { aut }_{1} X ; \mathbb{Q}\right) \cong \mathbb{Q}[a] \otimes \Lambda\left(b_{0}, b_{1}, b_{2}, \ldots\right) /\left(\left\{a b_{i}\right\}_{i},\left\{b_{i} b_{j}\right\}_{i<j}\right)
$$

with $|a|=4$ and $\left|b_{i}\right|=3+6 i$, which is not free.

\section{Pure case}

Definition 3.1 ([7]) An elliptic space $X$ is said to be pure if $d W^{\text {even }}=0$ and $d W^{\text {odd }} \subset$ $\Lambda W^{\text {even }}$ for $M(X)=(\Lambda W, d)$.

For example, a simply connected homogeneous space $G / H$ is a pure space. Of course, this class contains Lie groups. Also $F_{0}$-spaces are pure. The following lemma is an explicit description to apply Proposition 2.5 .

Lemma 3.2 For a $K S$-model $M(B)=\left(\Lambda V, d_{B}\right) \rightarrow(\Lambda V \otimes \Lambda W, D)$, the restriction $R_{n}: H_{n}\left(\operatorname{Der}_{\Lambda V}(\Lambda V \otimes \Lambda W), \delta\right) \rightarrow H_{n}(\operatorname{Der}(\Lambda W))$ is surjective if and only if there is an element $\rho$ of $\operatorname{Der}_{n}\left(\Lambda W, \Lambda^{+} V \otimes \Lambda W\right)$ such that $\theta+\rho$ is a non-exact $\delta$-cycle for any non-exact $\delta$-cycle $\theta$ of $\operatorname{Der}_{n}(\Lambda W)$.

Proposition 3.3 Let $X$ be a pure space with $\operatorname{rank} \pi_{\text {even }}(X)=2$. Then the followings are equivalent:

(i) $H_{\text {even }}(\operatorname{Der} M(X))=0$.

(ii) $R_{*}: H_{*}\left(\operatorname{Der}_{\Lambda V}(\Lambda V \otimes \Lambda W)\right) \rightarrow H_{*}(\operatorname{Der}(\Lambda W))=H_{*}(\operatorname{Der} M(X))$ is surjective for any $K S$-model $M\left(S^{n}\right)=\left(\Lambda V, d_{B}\right) \rightarrow(\Lambda V \otimes \Lambda W, D)$.

Proof Let $M(X)=(\Lambda W, d)=\left(\Lambda\left(x_{1}, x_{2}, y_{1}, . ., y_{m}\right), d\right)$ with $\left|x_{i}\right|$ even and $\left|y_{i}\right|$ odd. First, we show $(i i) \Rightarrow(i)$. Suppose $H_{\text {even }}(\operatorname{Der} M(X)) \neq 0$. Then, since $X$ is pure, there is an index $i$ such that

$$
\left[\left(y_{i}, f\right)+\cdots\right] \in H_{*}(\operatorname{Der} M(X))
$$


for some odd-degree element $f \in \mathbb{Q}\left[x_{1}, x_{2}\right] \otimes \Lambda^{1}\left(y_{1}, . ., y_{m}\right)$. Then we can construct a KS-model $(\Lambda v, 0) \rightarrow(\Lambda v \otimes \Lambda W, D)$ where $|v|=\left|y_{i}\right|-|f|+1$ is odd and

$$
D y_{i}=d y_{i}+f v
$$

(cf. [29]). Note that $f v$ is not cohomologous to an element of $\Lambda W$. (It is the KS-model of a rationally non-trivial fibration over an odd-sphere.) When $f$ contains $y_{j}(j<i)$, it follows from Lemma 3.2 that the element $\left[\left(y_{j}, 1\right)\right]$ of $H_{*}(\operatorname{Der} M(X))$ is not in the image of $R_{*}$. Because we have

$$
\delta\left(y_{j}, 1\right)= \pm\left(y_{i}, \partial f / \partial y_{j} \cdot v\right)+\cdots
$$

in $\operatorname{Der}_{\Lambda v}(\Lambda v \otimes \Lambda W)$, in which $\partial f / \partial y_{j} \cdot v$ is not $D$-exact. Then there is no derivation $\rho$ of $\operatorname{Der}\left(\Lambda W, \Lambda^{+} v \otimes \Lambda W\right)$ such that $\left(y_{j}, 1\right)+\rho$ is a $\delta$-cycle.

Next, we show (i) $\Rightarrow$ (ii). Since $X$ is pure, if $H_{\text {even }}(\operatorname{Der} M(X))=0$, then we get

$$
H_{*}(\operatorname{Der} M(X))=H_{*}(\operatorname{Der}(\Lambda W))=\oplus_{i=1}^{n} \mathbb{Q}\left\{\left[\left(y_{i}, f_{i j}\right)\right] ; f_{i j} \in \mathbb{Q}\left[x_{1}, x_{2}\right]\right\}
$$

Let $V=\mathbb{Q}\{v\}$ with $d_{B} v=0$ when $n$ is odd and $\mathbb{Q}\{v, z\}$ with $d_{B} z=v^{2}$ and $d_{B} v=0$ when $n$ is even. Then for any $\mathrm{KS}$-model $\left(\Lambda V, d_{B}\right) \rightarrow(\Lambda V \otimes \Lambda W, D)$, we can assume that the differential $D$ satisfies

$$
\begin{gathered}
D x_{i}=0 \text { and } D y_{i}=d y_{i}+\sum_{j} a_{i j} f_{i j} v \quad(|v|=n: \text { even }) \\
D x_{i}=0 \quad \text { and } D y_{i}=d y_{i} \quad(|v|=n: \text { odd })
\end{gathered}
$$

for some $a_{i j} \in \mathbb{Q}$ since $\operatorname{dim} W^{\text {even }}=2$. Thus it follows from Lemma 3.2 that $R_{*}$ is surjective, because each element $\left(y_{i}, f_{i j}\right)$ of $\operatorname{Der}(\Lambda W)$ is just a non-exact $\delta$-cycle in $\operatorname{Der}_{\Lambda V}(\Lambda V \otimes \Lambda W)$.

Theorem $1.1(1) \Leftrightarrow(2)$ follows from the geometrical realization of Proposition 3.3 as

Theorem 3.4 For a pure space $X$ with $\operatorname{rank} \pi_{\text {even }}(X)=2, H^{*}\left(B \operatorname{aut}_{1} X ; \mathbb{Q}\right)$ is a polynomial algebra if and only if $\pi_{*}(\operatorname{res} \xi)_{\mathbb{Q}}: \pi_{*}\left(\operatorname{aut}_{1} p\right)_{\mathbb{Q}} \rightarrow \pi_{*}\left(\operatorname{aut}_{1} X\right)_{\mathbb{Q}}$ is surjective for any fibration $\xi: E \stackrel{p}{\rightarrow} S^{n}$ with fiber $X$.

Proof It follows from Corollary 2.2 that Proposition 3.3 (i) is equivalent to $H^{*}\left(B\right.$ aut $\left._{1} X ; \mathbb{Q}\right)$ being a polynomial algebra, and it follows from Proposition 2.5 that Proposition 3.3 (ii) is equivalent to $\pi_{*}(\operatorname{res} \xi)_{\mathbb{Q}}: \pi_{*}\left(\operatorname{aut}_{1} p\right)_{\mathbb{Q}} \rightarrow \pi_{*}\left(\operatorname{aut}_{1} X\right)_{\mathbb{Q}}$ being surjective.

When $X$ is not an $F_{0}$-space, even if $H^{*}\left(B\right.$ aut $\left._{1} X ; \mathbb{Q}\right)$ is a polynomial algebra, there are non-tncz fibrations $\xi$. For example: 
Example 3.5 Let $X=S U(2) \times S U(2) \times S U(2) / T^{2}\left(T^{2}=S^{1} \times S^{1}\right)$, which is a homogeneous space space. Due to [26], there are two types of two-torus subgroups $T^{2} \subset S U(2) \times S U(2) \times S U(2)$ such that

$$
M(X)=\left(\Lambda\left(x_{1}, x_{2}, y_{1}, y_{2}, y_{3}\right), d\right) \text { with }\left|x_{1}\right|=\left|x_{2}\right|=2,\left|y_{1}\right|=\left|y_{2}\right|=\left|y_{3}\right|=3
$$

and

(1) $d x_{1}=d x_{2}=0, d y_{1}=x_{1}^{2}, d y_{2}=x_{2}^{2}, d y_{3}=0$ (formal [9]) and

(2) $d x_{1}=d x_{2}=0, d y_{1}=x_{1}^{2}, d y_{2}=x_{2}^{2}, d y_{3}=x_{1} x_{2}$ (non-formal).

In the case (1) there is a fibration $\xi: X \rightarrow E_{1} \rightarrow S^{4}$ such that $E_{1} \simeq_{\mathbb{Q}} S^{2} \times S^{2} \times S^{7}$ whose KS-model is given by $D y_{1}=d y_{1}, D y_{2}=d y_{2}$ and $D y_{3}=v$ for $H^{*}\left(S^{4} ; \mathbb{Q}\right)=$ $\mathbb{Q}[v] /\left(v^{2}\right)$.

In the case (2) there is a fibration $\xi: X \rightarrow E_{2} \rightarrow S^{4}$ with KS-model given by $D y_{3}=x_{1} x_{2}+v$. Then we have $H^{*}\left(E_{2} ; \mathbb{Q}\right)=\mathbb{Q}\left[x_{1}, x_{2}\right] /\left(x_{1}^{2}, x_{2}^{2}\right)$.

In both cases, $i^{*}: H^{*}\left(E_{j} ; \mathbb{Q}\right) \rightarrow H^{*}(X ; \mathbb{Q})$ are not surjective, since in the case (1) the element $\left[y_{3}\right]$ of $H^{3}(X ; \mathbb{Q})$ is not contained in $\operatorname{Im} i^{*}$ and in the case (2) the element $\left[x_{1} y_{3}-x_{2} y_{1}\right]$ of $H^{5}(X ; \mathbb{Q})$ is not contained in $\operatorname{Im} i^{*}$.

On the other hand, in both cases, we have that

$$
H^{*}\left(\text { Baut }_{1} X ; \mathbb{Q}\right)=\mathbb{Q}\left[u_{1}, u_{2}, u_{3}, u_{4}, u_{5}, u_{6}, u_{7}\right]
$$

with $\left|u_{1}\right|=\left|u_{2}\right|=\left|u_{3}\right|=\left|u_{4}\right|=2$ and $\left|u_{5}\right|=\left|u_{6}\right|=\left|u_{7}\right|=4$, since we have that

$$
\pi_{*}\left(\operatorname{aut}_{1} X\right)_{\mathbb{Q}}=\mathbb{Q}\left\{\left(y_{1}, x_{2}\right),\left(y_{2}, x_{k}\right),\left(y_{3}, x_{1}\right),\left(y_{3}, x_{2}\right),\left(y_{1}, 1\right),\left(y_{2}, 1\right),\left(y_{3}, 1\right)\right\},
$$

in which $k=1$ in the case (1) and $k=2$ in the case (2). Thus it follows from Theorem 3.4 (Theorem 1.1) that $\pi_{*}(\operatorname{res}(\xi))_{\mathbb{Q}}$ is surjective.

\section{Poset and depth}

Definition 4.1 Let $\xi$ and $\eta$ be two fibrations with fiber $X$ and base $B$. They are called $\mathbb{Q}$-fiber restriction equivalent (abbr. $\mathbb{Q}$-f.r.equivalent) if the images of the homomorphisms $\pi_{*}(\operatorname{res} \xi)_{\mathbb{Q}}$ and $\pi_{*}(\operatorname{res} \eta)_{\mathbb{Q}}$ are identical:

$$
\operatorname{Im}\left(\pi_{*}(\operatorname{res} \xi)_{\mathbb{Q}}\right)=\operatorname{Im}\left(\pi_{*}(\operatorname{res} \eta)_{\mathbb{Q}}\right) \subset \pi_{*}\left(\operatorname{aut}_{1} X\right)_{\mathbb{Q}} .
$$

Definition 4.2 For simply connected spaces $X$ and $B$, we define the poset $\mathcal{P}_{B}(X)$ to be the set of $\mathbb{Q}$-f.r.equivalence classes, denoted by double bracket $[[\xi]]$, of fibrations $\xi$ 's with fiber $X$ and base $B$ :

$$
\mathcal{P}_{B}(X):=(\{[[\xi]] \mid \xi: X \rightarrow E \rightarrow B \text { where } E \text { is finite }\}, \leq)
$$

in which

$$
[[\xi]] \leq[[\eta]] \Longleftrightarrow \operatorname{Im}\left(\pi_{n}(\operatorname{res} \xi)\right)_{\mathbb{Q}} \subset \operatorname{Im}\left(\pi_{n}(\operatorname{res} \eta)\right)_{\mathbb{Q}}, \forall n>0 .
$$


Then we define the depth of $X$ over $B$ as

$$
\operatorname{depth}_{B}(X):=\max \left\{n \mid\left[\left[\xi_{0}\right]\right]>\left[\left[\xi_{1}\right]\right]>\cdots>\left[\left[\xi_{n}\right]\right]\right\} .
$$

In particular, we denote $\operatorname{depth}_{B}(X)=-1$ when $\mathcal{P}_{B}(X)=\phi$. (For example, $\mathcal{P}_{K(\mathbb{Z}, 2)}\left(S^{2 n}\right)=\phi$, since $E$ cannot be finite in any fibration $S^{2 n} \rightarrow E \rightarrow K(\mathbb{Z}, 2)$.) Futhermore,

$$
\operatorname{depth}(X):=\sup \left\{\operatorname{depth}_{B}(X) \mid B \text { is simply connected }\right\}(\geq 0)
$$

Lemma 4.3 It holds that $\operatorname{depth}_{B}(X \times Y) \geq \max \left\{\operatorname{depth}_{B}(X)\right.$ depth $\left._{B}(Y)\right\}$. In particular, $\operatorname{depth}_{B}(X \times Y) \geq \operatorname{depth}_{B}(X)+\operatorname{depth}_{B}(Y)$ when $B=S^{2 n+1}$.

Proof The former is obvious since $M(X \times Y)=M(X) \otimes M(Y)$. The latter holds since the KS-model of any fibration over $S^{2 n+1}$ satisfies that $D W \subset \Lambda v \otimes \Lambda^{+} W$ for $M\left(S^{2 n+1}\right)=(\Lambda v, 0)$. Then we can construct the KS-model $(\Lambda v, 0) \rightarrow(\Lambda v \otimes$ $\left.\Lambda W \otimes \Lambda U, D^{\prime \prime}\right)$ with $\left.D^{\prime \prime}\right|_{W}=D$ and $\left.D^{\prime \prime}\right|_{U}=D^{\prime}$ for any $\mathrm{KS}$-models $(\Lambda v, 0) \rightarrow$ $(\Lambda v \otimes \Lambda W, D)$ and $(\Lambda v, 0) \rightarrow\left(\Lambda v \otimes \Lambda U, D^{\prime}\right)$.

Let $[[11]]$ denote the $\mathbb{Q}$-f.r.equivalence class of the trivial fibration $\mathbb{1 1}$ with fiber $X$ and finite base space $B$, thus $\operatorname{Im}\left(\pi_{*}(\operatorname{res} 1)\right)_{\mathbb{Q}}=\pi_{*}\left(\text { aut }_{1} X\right)_{\mathbb{Q}}$. Hence for any fibration $\xi$ we have that $[[\xi]] \leq[[11]]$, i.e., $[[11]]$ is the maximal point of the poset $\mathcal{P}_{B}(X)$. Hence the following lemma is clear.

Lemma 4.4 $\pi_{*}(\operatorname{res} \xi)_{\mathbb{Q}}$ is surjective for any fibration $\xi: X \rightarrow E \rightarrow B$ over a finite base $B$ if and only if $\operatorname{depth}_{B}(X)=0$.

Hence we deduce the equivalence $(2) \Leftrightarrow$ (3) of Theorem 1.1.

Even if $\operatorname{rank} G>\operatorname{rank} H$, it may hold that $\operatorname{depth}(G / H)=0$. For example, see Example 3.5, in which $\operatorname{rank} G=3>2=\operatorname{rank} H$. Futhermore we have obviously

Lemma 4.5 When $X \simeq{ }_{\mathbb{Q}} S^{n_{1}} \times \cdots \times S^{n_{m}}$ with all $n_{i}$ odd, $\operatorname{depth}(X)=0$ if and only if $n_{1}=\ldots=n_{m}$. In particular, $\operatorname{depth}\left(S^{n}\right)=0$ for any $n>1$.

See $\S 5$ for the examples such that $n_{1}, \ldots, n_{m}$ are not equal.

Remark 4.6 Let $\mathcal{F}_{B}(X):=$ \{fibration $\xi$ over $B$ with fibre $\left.X\right\} / \simeq$ fhe, where $\simeq$ fhe denotes fibre-homotopy equivalence [5]. When $B$ is finite, there is the following surjection

$$
\Phi: \mathcal{F}_{B}(X) \rightarrow \mathcal{P}_{B}(X)
$$

given by $\Phi([\xi])=[[\xi]]$. Of course, it is not bijective in general. For example, $\Phi^{-1}([[\xi]])$ can be infinite (see Example 4.7 below).

Example 4.7 There are fibrations $S^{3} \rightarrow E_{a} \rightarrow B$ with infinitely many different rational homotopy type $E_{a}$ over $B$ with

$M(B)=\left(\Lambda\left(v_{1}, v_{2}, v\right), d\right)$ with $\left|v_{2}\right|=\left|v_{2}\right|=2,|v|=3, d v_{1}=d v_{2}=0, d v=v_{1} v_{2}$ 
When a fibration $\xi_{a}$ for $a \in \mathbb{Q}^{*}=\mathbb{Q}-\{0\}$ is given by the KS-model

$$
\begin{aligned}
& \left(\Lambda\left(v_{1}, v_{2}, v\right), d\right) \rightarrow\left(\Lambda\left(v_{1}, v_{2}, v, w\right), D_{a}\right) \text { with }|w|=3, \\
& D_{a} v=v_{1} v_{2}, D_{a} w=v_{1}^{2}+a v_{2}^{2},
\end{aligned}
$$

$E_{a}$ 's are all rational-homotopically different for each $a \in \mathbb{Q}^{*} /\left(\mathbb{Q}^{*}\right)^{2}$, although $\xi_{a}$ 's are all $\mathbb{Q}$-f.r.equivalent to each other, since $\pi_{*}\left(\operatorname{aut}_{1} S^{3}\right) \mathbb{Q}=\operatorname{Im} \pi_{*}\left(\operatorname{res} \xi_{a}\right) \mathbb{Q} \cong \mathbb{Q}$ for all a.

Remark 4.8 Recall that the depth of the rational Gottlieb poset of $X$ over $B$ in [33]. Denote it as depth ${ }_{B}^{G}(X)$ in this paper. From the proof of Proposition 3.3 with Theorem 2.3, we can deduce that $\operatorname{depth}_{S^{n}}(X)=0$ for any $n$ if and only if $\operatorname{depth}_{S^{n}}^{G}(X)=0$ for any $n$ when $X$ is pure with $\operatorname{rank} \pi_{e v e n}(X)=2$.

\section{Examples}

In this section, all KS-models are realized as fibrations in which the total spaces are finite.

As we recalled the definition of the fibre restricted Gottlieb group $G_{*}^{\xi}(X)$ for a fibration $\xi$ ([33]) in the introduction, even if $G_{*}(X)_{\mathbb{Q}}=G_{*}^{\xi}(X)_{\mathbb{Q}}$, it of course may happen that $\pi_{*}\left(\operatorname{aut}_{1} X\right)_{\mathbb{Q}} \neq \operatorname{Im} \pi_{*}(\operatorname{res} \xi)_{\mathbb{Q}}$, as shown in the following example:

Example 5.1 Let $M(X)=\left(\Lambda\left(w_{1}, w_{2}, w_{3}, w_{4}, w\right), d\right)$ with

$d w_{i}=0, d w=w_{1} w_{2} w_{3} w_{4},\left|w_{1}\right|=\left|w_{2}\right|=3,\left|w_{3}\right|=5,\left|w_{4}\right|=7$ and $|w|=17$.

Then, if $D w_{3}=v w_{1}$ (and $D=d$ for the others) for a KS-model

$$
(\Lambda v, 0) \rightarrow\left(\Lambda v \otimes \Lambda\left(w_{1}, w_{2}, w_{3}, w_{4}, w\right), D\right) \text { with }|v|=3
$$

it follows from Lemma 3.2 that the element $\left(w_{4}, w_{3}\right)$ of $H_{2}(\operatorname{Der} M(X))$ is not contained in $\operatorname{Im} R_{2}$. Thus from Proposition 2.5 we get the inequality $\pi_{*}\left(\operatorname{aut}_{1} X\right)_{\mathbb{Q}} \neq$ $\operatorname{Im} \pi_{*}(\operatorname{res} \xi)_{\mathbb{Q}}$. On the other hand it follows from Theorem 2.3 that $G_{*}(X)_{\mathbb{Q}}=$ $G_{*}^{\xi}(X)_{\mathbb{Q}} \cong \mathbb{Q}\{(w, 1)\}$.

Example 5.2 Let us recall that the exceptional simple Lie group $E_{6}$ has the following rational homotopy type:

$$
E_{6} \simeq \mathbb{Q} S^{3} \times S^{9} \times S^{11} \times S^{15} \times S^{17} \times S^{23} .
$$

Then the three depths depth $S^{3}\left(E_{6}\right)$, depth $S^{4}\left(E_{6}\right)$ and depth $S^{5}\left(E_{6}\right)$ are all different as follows:

(1) $\operatorname{depth}_{S^{3}}\left(E_{6}\right)=2$

(2) $\operatorname{depth}_{S^{4}}\left(E_{6}\right)=3$

(3) $\operatorname{depth}_{S^{5}}\left(E_{6}\right)=1$. 
Indeed, let $M(X)=\left(\Lambda\left(w_{1}, w_{2}, w_{3}, w_{4}, w_{5}, w_{6}\right), 0\right)$ with

$$
\left|w_{1}\right|=3,\left|w_{2}\right|=9,\left|w_{3}\right|=11,\left|w_{4}\right|=15,\left|w_{5}\right|=17,\left|w_{6}\right|=23
$$

and $H^{*}\left(S^{n} ; \mathbb{Q}\right)=\Lambda(v) /\left(v^{2}\right)$. Then (1), (2) and (3) are given by the following chains of KS-models with the maximal length:

(1) $[[11]]>\left[\left[\xi_{1}\right]\right]>\left[\left[\xi_{2}\right]\right]$ with

$$
\begin{aligned}
& \xi_{1}: D w_{1}=D w_{2}=D w_{3}=D w_{4}=0, D w_{5}=v w_{4}, D w_{6}=0 \\
& \xi_{2}: D w_{1}=D w_{2}=0, D w_{3}=v w_{2}, D w_{4}=0, D w_{5}=v w_{4}, D w_{6}=0,
\end{aligned}
$$

(2) $[[11]]>\left[\left[\xi_{1}\right]\right]>\left[\left[\xi_{2}\right]\right]>\left[\left[\xi_{3}\right]\right]$ with

$\xi_{1}: D w_{1}=D w_{2}=D w_{3}=0, D w_{4}=v w_{1} w_{2}, D w_{5}=D w_{6}=0$

$\xi_{2}: D w_{1}=D w_{2}=D w_{3}=0, D w_{4}=v w_{1} w_{2}, D w_{5}=v w_{1} w_{3}, D w_{6}=0$

$\xi_{3}: D w_{1}=D w_{2}=D w_{3}=0, D w_{4}=v w_{1} w_{2}, D w_{5}=v w_{1} w_{3}, D w_{6}=$ $v w_{1} w_{5}$ and

(3) $[[11]]>\left[\left[\xi_{1}\right]\right]$ with

$\xi_{1}: D w_{1}=D w_{2}=D w_{3}=0, D w_{4}=v w_{3}, D w_{5}=D w_{6}=0$.

Example 5.3 As to the special unitary group $S U(n)$, for $n>1$, we have

$$
\operatorname{depth}_{S^{3}}(S U(n)) \geq n-1 \text {. }
$$

Indeed, let $M(S U(n))=\left(\Lambda\left(w_{1}, w_{2}, \ldots w_{n-1}\right), 0\right)$ with

$$
\left|w_{1}\right|=3,\left|w_{2}\right|=5, \ldots,\left|w_{n-1}\right|=2 n-1
$$

and $M\left(S^{3}\right)=(\Lambda(v), 0)$. When $\xi_{k}(k>1)$ is given by the KS-model

$$
(\Lambda(v), 0) \rightarrow\left(\Lambda\left(v, w_{1}, w_{2}, \ldots w_{n-1}\right), D_{k}\right)
$$

with the differential $D_{k}$ defined by:

$$
D_{k} w_{1}=0, D_{k} w_{2}=v w_{1}, \ldots, D_{k} w_{k}=v w_{k-1}, D_{k} w_{k+1}=\ldots=D_{k} w_{n-1}=0,
$$

then we have that $\operatorname{Im} \pi_{i}\left(\operatorname{res}\left(\xi_{k}\right)\right)_{\mathbb{Q}}=\operatorname{Der}_{i}\left(\Lambda\left(w_{k}, \ldots, w_{n-1}\right)\right)$ for all $i$. Thus we have

$$
\left[\left[\xi_{n-1}\right]\right]<\left[\left[\xi_{n-2}\right]\right]<\cdots<\left[\left[\xi_{2}\right]\right]<[[11]]
$$

Example 5.4 (1) Let $X$ be a homogeneous space with $X=S U(6) / S U(3) \times S U(3)$. Then we have depth $(X) \neq 0$. Especially

$$
\operatorname{depth}_{S^{3}}(X) \geq 1
$$

Indeed, let $M(X)=\left(\Lambda\left(w_{1}, w_{2}, w_{3}, w_{4}, w_{5}\right), d\right)$ with $\left|w_{1}\right|=4,\left|w_{2}\right|=6,\left|w_{3}\right|=$ $7,\left|w_{4}\right|=9,\left|w_{5}\right|=11, d w_{1}=d w_{2}=0, d w_{3}=w_{1}^{2}, d w_{4}=w_{1} w_{2}$ and $d w_{5}=w_{2}^{2}$ (see [15]). Then, it follows from the isomorphism (2.1) in $\S 2$ that

$$
\pi_{*}\left(\operatorname{aut}_{1} X\right)_{\mathbb{Q}} \cong H_{*}(\operatorname{Der} M(X))
$$


$\cong \mathbb{Q}\left\{\left(w_{5}, w_{1}\right),\left(w_{5}, w_{2}\right),\left(w_{3}, w_{1}\right),\left(w_{3}, w_{2}\right),\left(w_{3}, 1\right),\left(w_{4}, 1\right),\left(w_{5}, 1\right)\right.$,

$$
\left.2\left(w_{5}, w_{4}\right)+\left(w_{4}, w_{3}\right)+\left(w_{2}, w_{1}\right)\right\}
$$

Let $\xi$ be a fibration given by the KS-model with differential $D$ defined by:

$D w_{1}=0, D w_{2}=v w_{1}, D w_{3}=w_{1}^{2}, D w_{4}=w_{1} w_{2}-v w_{3}, D w_{5}=w_{2}^{2}-2 v w_{4}$

Then it follows from Proposition 2.5 that

$\operatorname{Im} \pi_{*}(\operatorname{res}(\xi))_{\mathbb{Q}} \cong \mathbb{Q}\left\{\left(w_{5}, w_{1}\right),\left(w_{5}, 1\right), 2\left(w_{5}, w_{4}\right)+\left(w_{4}, w_{3}\right)+\left(w_{2}, w_{1}\right)\right\}$

We recall that

$$
M\left(B \operatorname{aut}_{1} X\right)=\left(\Lambda\left(v_{2}, v_{3}, v_{4}, v_{6}, v_{8}, v_{8}^{\prime}, v_{10}, v_{12}\right), d\right)
$$

with $\left|v_{n}\right|=\left|v_{n}^{\prime}\right|=n, d v_{2}=d v_{3}=d v_{8}=0, d v_{4}=v_{2} v_{3}, d v_{6}=v_{4} v_{3}$, $d v_{8}^{\prime}=v_{6} v_{3}, d v_{10}=v_{8} v_{3}$ and $d v_{12}=v_{10} v_{3}$ [25]. In particular, $H^{*}\left(B \operatorname{aut}_{1} X ; \mathbb{Q}\right)$ is not free.

(2) Let $X$ be a homogeneous space with $X=S U(7) / S U(3) \times S U(4)$. From [1, Proposition 2.8], $M(X)=\left(\Lambda\left(w_{1}, w_{2}, w_{3}, w_{4}, w_{5}\right), d\right)$ with $\left|w_{1}\right|=4,\left|w_{2}\right|=6$, $\left|w_{3}\right|=9,\left|w_{4}\right|=11,\left|w_{5}\right|=13$ and $d w_{1}=d w_{2}=0, d w_{3}=w_{1} w_{2}, d w_{4}=$ $w_{1}^{3}-w_{2}^{2}, d w_{5}=0$ (formal). Then $H^{*}\left(B\right.$ aut $\left._{1} X ; \mathbb{Q}\right)$ is a polynomial algebra [25]. Therefore $\operatorname{depth}_{S^{n}}(X)=0$ for any $n$ from Theorem 1.1 .

(3) Let $X$ be a homogeneous space with $X=S U(8) / S U(3) \times S U(5)$. From [1, Appendix A], we see that $M(X)=\left(\Lambda\left(w_{1}, w_{2}, w_{3}, w_{4}, w_{5}\right), d\right)$ with $\left|w_{1}\right|=$ $4,\left|w_{2}\right|=6,\left|w_{3}\right|=11,\left|w_{4}\right|=13,\left|w_{5}\right|=15$ and $d w_{1}=d w_{2}=0, d w_{3}=$ $w_{1}^{3}-w_{2}^{2}, d w_{4}=w_{1}^{2} w_{2}, d w_{5}=w_{1} w_{2}^{2}$ (non-formal). Then $H^{*}\left(B\right.$ aut $\left._{1} X ; \mathbb{Q}\right)$ is a polynomial algebra [25]. Therefore $\operatorname{depth}_{S^{n}}(X)=0$ for any $n$ from Theorem 1.1.

Example 5.5 (1) Let $X_{1}=S U(6) \simeq \mathbb{Q} S^{3} \times S^{5} \times S^{7} \times S^{9} \times S^{11}, B=K(\mathbb{Z}, 2)$ and let $M\left(X_{1}\right)=(\Lambda W, 0)=\left(\Lambda\left(w_{1}, w_{2}, w_{3}, w_{4}, w_{5}\right), 0\right)$ with

$$
\left|w_{1}\right|=3,\left|w_{2}\right|=5,\left|w_{3}\right|=7,\left|w_{4}\right|=9,\left|w_{5}\right|=11
$$

and $M(B)=(\mathbb{Q}[t], 0)$ with $|t|=2$.

Then the equivalence classes of $\mathcal{P}_{B}\left(X_{1}\right)$ are given by the following KS-models:

(a) $D w_{1}=D w_{2}=D w_{3}=D w_{4}=0, D w_{5}=t^{6}$

(b) $D w_{1}=D w_{2}=D w_{3}=D w_{4}=0, D w_{5}=w_{1} w_{3} t+t^{6}$

(c) $D w_{1}=D w_{2}=D w_{3}=D w_{4}=0, D w_{5}=w_{1} w_{2} t^{3}+t^{6}$

(d) $D w_{1}=D w_{2}=D w_{3}=0, D w_{4}=w_{1} w_{2} t, D w_{5}=t^{6}$

(e) $D w_{1}=D w_{2}=D w_{3}=0, D w_{4}=w_{1} w_{2} t, D w_{5}=w_{1} w_{3} t+t^{6}$ 
Futhermore the bases of $\operatorname{Im}\left(R_{*}: H_{*}\left(\operatorname{Der}_{\mathbb{Q}[t]}(\mathbb{Q}[t] \otimes \Lambda W)\right) \rightarrow H_{*}(\operatorname{Der}(\Lambda W))\right)$ are given by the following table:

\begin{tabular}{llllll}
\hline$n$ & $(a)$ & $(b)$ & $(c)$ & $(d)$ & $(e)$ \\
\hline 1 & $\left(w_{5}, w_{1} w_{3}\right)$ & $\left(w_{5}, w_{1} w_{3}\right)$ & $\left(w_{5}, w_{1} w_{3}\right)$ & $\left(w_{5}, w_{1} w_{3}\right)$ & $\left(w_{5}, w_{1} w_{3}\right)$ \\
2 & $w_{5,4}, w_{4,3}$, & $w_{5,4}, w_{4,3}$, & $w_{5,4}, w_{4,3}, w_{3,2}$, & $w_{4,3}, w_{3,2}$, & $w_{4,3}, w_{3,2}$, \\
& $w_{3,2}, w_{2,1}$ & $w_{2,1}$ & $w_{2,1}$ & $w_{2,1}$ & $w_{2,1}$ \\
3 & $\left(w_{1}, 1\right)$, & $\left(w_{5}, w_{1} w_{2}\right)$ & $\left(w_{5}, w_{1} w_{2}\right)$ & $\left(w_{5}, w_{1} w_{2}\right)$ & $\left(w_{5}, w_{1} w_{2}\right)$ \\
& $\left(w_{5}, w_{1} w_{2}\right)$ & & & & \\
4 & $w_{5,3}$, & $w_{5,3}, w_{4,2}$, & $w_{5,3}$, & $w_{5,3}, w_{4,2}$, & $w_{5,3}, w_{4,2}$, \\
& $w_{4,2}, w_{3,1}$ & $w_{3,1}$ & $w_{4,2}, w_{3,1}$ & $w_{3,1}$ & $w_{3,1}$ \\
5 & $\left(w_{2}, 1\right)$ & $\left(w_{2}, 1\right)$ & & & $\left(w_{5}, w_{2}\right)$, \\
6 & $\left(w_{5}, w_{2}\right)$, & $\left(w_{5}, w_{2}\right)$, & $\left(w_{5}, w_{2}\right)$, & $\left(w_{5}, w_{2}\right)$, \\
7 & $\left(w_{4}, w_{1}\right)$ & $\left(w_{4}, w_{1}\right)$ & $\left(w_{4}, w_{1}\right)$ & $\left(w_{4}, w_{1}\right)$ & $\left(w_{4}, w_{1}\right)$ \\
8 & $\left(w_{3}, 1\right)$ & & $\left(w_{3}, 1\right)$ & $\left(w_{3}, 1\right)$ & $\left(w_{5}, w_{1}\right)$ \\
9 & $\left(w_{5}, w_{1}\right)$ & $\left(w_{5}, w_{1}\right)$ & $\left(w_{5}, w_{1}\right)$ & $\left(w_{4}, 1\right)$ & $\left(w_{1}\right)$ \\
10 & $\left(w_{4}, 1\right)$ & $\left(w_{4}, 1\right)$ & $\left(w_{4}, 1\right)$ & & $\left(w_{4}, 1\right)$ \\
11 & $\left(w_{5}, 1\right)$ & $\left(w_{5}, 1\right)$ & $\left(w_{5}, 1\right)$ & $\left(w_{5}, 1\right)$ & \\
\hline
\end{tabular}

where $w_{i, j}:=\left(w_{i}, w_{j}\right)$

(2) Let $X_{2}=S^{3} \times S^{5} \times S^{9} \times S^{19}, B=K(\mathbb{Z}, 2)$ and let $M\left(X_{2}\right)=(\Lambda W, 0)=$ $\left(\Lambda\left(w_{1}, w_{2}, w_{3}, w_{4}\right), 0\right)$ with

$$
\left|w_{1}\right|=3,\left|w_{2}\right|=5,\left|w_{3}\right|=9,\left|w_{4}\right|=19 .
$$

From the degree reason, we can set as

$$
\begin{gathered}
D w_{1}=D w_{2}=0, D w_{3}=a_{1} w_{1} w_{2} t+a_{2} t^{5} \\
D w_{4}=a_{3} w_{1} w_{2} t^{6}+a_{4} w_{1} w_{3} t^{4}+a_{5} w_{2} w_{3} t^{3}+a_{6} t^{10}
\end{gathered}
$$

for $a_{1}, . ., a_{6} \in \mathbb{Q}$, in general. Then the equivalence classes of $\mathcal{P}_{B}\left(X_{2}\right)$ are given by the following KS-models:

(a) $D w_{1}=D w_{2}=D w_{3}=0, D w_{4}=t^{10}$

(b) $D w_{1}=D w_{2}=D w_{3}=0, D w_{4}=w_{2} w_{3} t^{3}+t^{10}$

(c) $D w_{1}=D w_{2}=D w_{3}=0, D w_{4}=w_{1} w_{3} t^{4}+t^{10}$

(d) $D w_{1}=D w_{2}=D w_{4}=0, D w_{3}=w_{1} w_{2} t, D w_{4}=t^{10}$

(e) $D w_{1}=D w_{2}=0, D w_{3}=w_{1} w_{2} t+t^{5}, D w_{4}=0$

(f) $D w_{1}=D w_{2}=0, D w_{3}=w_{1} w_{2} t, D w_{4}=w_{1} w_{3} t^{4}+t^{10}$

(g) $D w_{1}=D w_{2}=0, D w_{3}=w_{1} w_{2} t, D w_{4}=w_{2} w_{3} t^{3}+t^{10}$

Futhermore the bases of $\operatorname{Im}\left(R_{*}: H_{*}\left(\operatorname{Der}_{\mathbb{Q}[t]}(\mathbb{Q}[t] \otimes \Lambda W)\right) \rightarrow H_{*}(\operatorname{Der}(\Lambda W))\right)$ are given by the following table:

Their Hasse diagrams are given as follows: 


\begin{tabular}{lllll}
\hline$n$ & $(a)$ & $(b)$ & $(c)$ & $(d)$ \\
\hline 1 & $\left(w_{3}, w_{1} w_{2}\right)$ & $\left(w_{3}, w_{1} w_{2}\right)$ & $\left(w_{3}, w_{1} w_{2}\right)$ & $\left(w_{3}, w_{1} w_{2}\right)$ \\
2 & $\left(w_{4}, w_{123}\right),\left(w_{2}, w_{1}\right)$ & $\left(w_{4}, w_{123}\right)$ & $\left(w_{4}, w_{123}\right),\left(w_{2}, w_{1}\right)$ & $\left(w_{4}, w_{123}\right),\left(w_{2}, w_{1}\right)$ \\
3 & $\left(w_{1}, 1\right)$ & $\left(w_{1}, 1\right)$ & & \\
4 & $\left(w_{3}, w_{2}\right)$ & $\left(w_{3}, w_{2}\right)$ & $\left(w_{3}, w_{2}\right)$ & $\left(w_{3}, w_{2}\right)$ \\
5 & $\left(w_{2}, 1\right),\left(w_{4}, w_{2} w_{3}\right)$ & $\left(w_{4}, w_{2} w_{3}\right)$ & $\left(w_{2}, 1\right),\left(w_{4}, w_{2} w_{3}\right)$ & $\left(w_{4}, w_{2} w_{3}\right)$ \\
6 & $\left(w_{3}, w_{1}\right)$ & & $\left(w_{3}, w_{1}\right)$ & $\left(w_{3}, w_{1}\right)$ \\
7 & $\left(w_{4}, w_{1} w_{3}\right)$ & $\left(w_{4}, w_{1} w_{3}\right)$ & $\left(w_{4}, w_{1} w_{3}\right)$ & $\left(w_{4}, w_{1} w_{3}\right)$ \\
9 & $\left(w_{3}, 1\right)$ & $\left(w_{3}, 1\right)$ & $\left(w_{4}, w_{1} w_{2}\right)$ \\
10 & $\left(w_{4}, w_{3}\right)$ & $\left(w_{4}, w_{1} w_{2}\right)$ & $\left(w_{4}, w_{1} w_{2}\right)$ & $\left(w_{4}, w_{2}\right)$ \\
11 & $\left(w_{4}, w_{1} w_{2}\right)$ & $\left(w_{4}, w_{2}\right)$ & $\left(w_{4}, w_{2}\right)$ & $\left(w_{4}, w_{1}\right)$ \\
14 & $\left(w_{4}, w_{2}\right)$ & $\left(w_{4}, w_{1}\right)$ & $\left(w_{4}, w_{1}\right)$ & $\left(w_{4}, 1\right)$ \\
16 & $\left(w_{4}, w_{1}\right)$ & $\left(w_{4}, 1\right)$ & $\left(w_{4}, 1\right)$ & \\
19 & $\left(w_{4}, 1\right)$ & & & \\
\hline
\end{tabular}

\begin{tabular}{llll}
\hline$n$ & $(e)$ & $(f)$ & $(g)$ \\
\hline 1 & $\left(w_{3}, w_{1} w_{2}\right)$ & $\left(w_{3}, w_{1} w_{2}\right)$ & $\left(w_{3}, w_{1} w_{2}\right)$ \\
2 & $\left(w_{2}, w_{1}\right)$ & $\left(w_{4}, w_{123}\right),\left(w_{2}, w_{1}\right)$ & $\left(w_{2}, w_{123}\right)$ \\
3 & & & \\
4 & $\left(w_{3}, w_{2}\right)$ & $\left(w_{3}, w_{2}\right)$ & $\left(w_{3}, w_{2}\right)$ \\
5 & & $\left(w_{4}, w_{2} w_{3}\right)$ & $\left(w_{4}, w_{2} w_{3}\right)$ \\
6 & $\left(w_{3}, w_{1}\right)$ & $\left(w_{3}, w_{1}\right)$ & $\left(w_{1}\right)$ \\
7 & $\left(w_{4}, w_{1} w_{3}\right)$ \\
9 & $\left(w_{3}, 1\right)$ & $\left(w_{4}, w_{1} w_{3}\right)$ & \\
10 & $\left(w_{4}, w_{1} w_{2}\right)$ & & $\left(w_{4}, w_{1} w_{2}\right)$ \\
11 & $\left(w_{4}, w_{2}\right)$ & $\left(w_{4}, w_{1} w_{2}\right)$ & $\left(w_{4}, w_{2}\right)$ \\
14 & $\left(w_{4}, w_{1}\right)$ & $\left(w_{4}, w_{2}\right)$ & $\left(w_{4}, w_{1}\right)$ \\
16 & $\left(w_{4}, 1\right)$ & $\left(w_{4}, w_{1}\right)$ & $\left(w_{4}, 1\right)$ \\
19 & $\left(w_{4}, 1\right)$ & \\
\hline
\end{tabular}

where $w_{123}:=w_{1} w_{2} w_{3}$

(1)

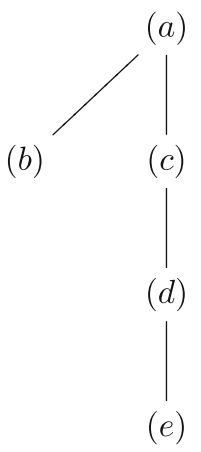

(2)

(b)

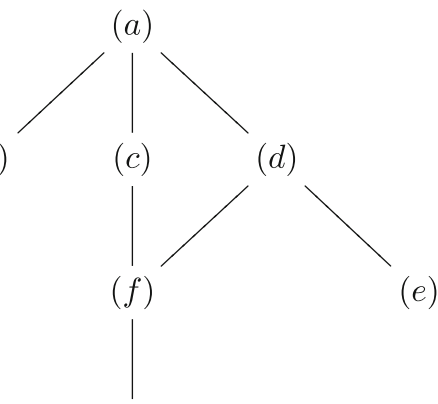

(g)

Thus we have that

$$
\operatorname{depth}_{K(\mathbb{Z}, 2)}\left(X_{1}\right)=\operatorname{depth}_{K(\mathbb{Z}, 2)}\left(X_{2}\right)=3 .
$$


However, we have that

$$
r_{0}\left(X_{1}\right)=5>4=r_{0}\left(X_{2}\right),
$$

where $r_{0}\left(X_{i}\right)$ means the rational toral rank of $X_{i}$ [16]. From the above results, we see that there are rationally 5 types of almost free $S^{1}$-actions on spaces which are rationally equivalent to $X_{1}$ and 7 types of almost free $S^{1}$-actions on spaces which are rationally equivalent to $X_{2}$.

Acknowledgements The second author has been supported in part by JSPS KAKENHI Grant Number $23244008,24540085,16 \mathrm{H} 03936$.

\section{References}

1. Amann, M.: Non-formal homogeneous spaces. Math. Z. 274, 1299-1325 (2013)

2. Amann, M., Kapovitch, V.: On fibrations with formal elliptic fibers. Adv. Math. 231, 2048-2068 (2012)

3. Borel, A.: Sur la cohomolgie des espaces fibrés principauz et des espaces homogènes de groups de Lie compacts. Ann. Math. 57, 115-207 (1953)

4. Borel, A.: Cohomologie des espaces localement compacts d'aprés. J. Leray. Springer, Lecture Nores Math 2 (1964)

5. Buijs, U., Smith, S.B.: Rational homotopy type of the classifying space for fibrewise self-equivalences. Proc. Am. Math. Soc. 141, 2153-2167 (2013)

6. Dold, A., Lashof, R.: Principal quasi-fibrations and fibre homotopy equivalence of bundles. Ill. J. Math. 3, 285-305 (1959)

7. Félix, Y.: Dénombrement des types de K-homotopie théorie de la déformation. Mem. Soc. Math. Fr. 3, 1-49 (1980)

8. Félix, Y., Halperin, S.: Rational LS category and its applications. Trans. Am. Math. Soc. 273, 1-38 (1982)

9. Félix, Y., Halperin, S., Thomas, J.C.: Rational homotopy theory, vol. 205. Springer, Berlin (2001)

10. Félix, Y., Lupton, G., Smith, S.B.: The rational homotopy type of the space of self-equivalences of a fibration. Homol Homot Appl 12(2), 371-400 (2010)

11. Félix, Y., Oprea, J.: Rational homotopy of gauge groups. Proc. Am. Math. Soc. 137, 1519-1527 (2009)

12. Gottlieb, D.H.: On fibre spaces and the evaluation map. Ann. Math. 87, 42-55 (1968)

13. Gottlieb, H.: Evaluation subgroups of homotopy groups. Am. J. Math. 91, 729-756 (1969)

14. Gottlieb, H.: Applications of bundle map theory. Trans. Am. Math. Soc. 171, 23-50 (1972)

15. Greub, W., Halperin, S., Vanstone, R.: Connection, curvature and cohomology III. Academic press, Dublin (1976)

16. Halperin, S.: Rational homotopy and torus actions. Lond. Math. Soc. Lecture note series, vol. 93, pp. 293-306. Cambridge Univ. Press, Cambridge (1985)

17. Hamada, K., Yamaguchi, T., Yokura, S.: C-symplectic poset structure on a simply connected space. Topol. Appl. 161, 107-126 (2014)

18. Hirsch, G.: Un isomorphisme attaché aux structures fibré. C. R. Acad. Sci. Paris 227, 1328-1330 (1948)

19. Hilton, P., Mislin, G., Roitberg, J.: Localization of nilpotent groups and spaces. N.-Holl. Math. Stud. 15 (1975)

20. Leray, J.: L'homologie d'un espace fibr $d$ ont la fibre estconnexe. J. Math. Pures Appl. 29, 169-213 (1950)

21. Lupton, G.: Variations on a conjecture of Halperin. Homotopy and geometry, vol. 45, pp. 115-135. Banach Centre Publications, Warszawa (1998)

22. Meier, W.: Rational universal fibrations and flag manifolds. Math. Ann. 258, 329-340 (1983)

23. McClearly, J.: A user's guide to spectral sequences. Cambridge Stud. Adv. Math., vol. 58, 2nd edn. Cambridge Univ. Press, Cambridge (2001)

24. Nishinobu, H., Yamaguchi, T.: Certain examples of posets of rational Gottlieb subgroups. Kochi Math. J. 10, 77-94 (2015) 
25. Nishinobu, H., Yamaguchi, T.: Sullivan minimal models of classifying spaces for non-formal spaces of small rank. Topol. Appl. 196, 290-307 (2015)

26. Parent, P.-E.: Formal and non-formal homogeneous spaces of small rank. Thesis, Univ. of Ottawa (1996)

27. Salvatore, P.: Rational homotopy nilpotency of self-equivalences. Topology and its Appl. 77, 37-50 (1997)

28. Serre, J.P.: Homologie singuliére des espaces fibrés. Appl. Ann. Math. 54, 425-505 (1951)

29. Schlessinger, M., Stasheff, J.: Deformation theory and rational homotopy type. arXiv:1211.1647v1

30. Shiga, H., Tezuka, M.: Rational fibrations, homogeneous spaces with positive Euler characteristics and Jacobians. Ann. Inst. Fourier (Grenoble) 37, 81-106 (1987)

31. Sullivan, D.: Infinitesimal computations in topology. I.H.E.S. 47, 269-331 (1978)

32. Tanré, D.: Homotopie Rationnelle: Modèles de Chen, Quillen, Sullivan. Lecture Note in Nath. Springer, Berlin (1983)

33. Yamaguchi, T.: A fibre-restricted Gottlieb group and its rational realization problem. Topol. Appl. 196, 1060-1076 (2015) 